Trinity University

Digital Commons @ Trinity

Philosophy Faculty Research

Philosophy Department

1992

\title{
The Sounds of Music: First Movement
}

Lawrence Kimmel

Trinity University, Ikimmel@trinity.edu

Follow this and additional works at: https://digitalcommons.trinity.edu/phil_faculty

Part of the Philosophy Commons

\section{Repository Citation}

Kimmel, L. (1992). The sounds of music: First movement. Journal of Aesthetic Education, 26(3), 55-65. doi:10.2307/3333013

This Post-Print is brought to you for free and open access by the Philosophy Department at Digital Commons @ Trinity. It has been accepted for inclusion in Philosophy Faculty Research by an authorized administrator of Digital Commons@ Trinity. For more information, please contact jcostanz@trinity.edu. 


\title{
The Sounds of Music: First Movement
}

\author{
LAWRENCE D. KIMMEL
}

How we hear music clearly depends upon our intellectual capacities and education, upon concepts, analogies and expectations that we have inherited from a culture steeped in musical expression. Yet the understanding that we derive from this culture is manifest in our way of hearing, and not just our way of thinking about music.

-Roger Scruton

In his essay "Understanding Music," Roger Scruton has argued for a nonreductionist approach to aesthetics, emphasizing the contextually rich language and grammar that structure discourse about various forms of art. This accords with Wittgenstein's series of "reminders" about the importance of finding philosophical footholds in ordinary language. However refined artistic taste and aesthetic judgment may become, their fundamental source is in ordinary discourse about what surprises, pleases, and moves us. In what follows I will try to amplify these remarks and map the outlines of a conceptual investigation of the grammar of musical understanding. I will be less interested in an account of how we hear music than in an examination of the idea that we hear music.

There are of course many kinds and degrees of "understanding." In music, as elsewhere, understanding may and does result from study, analysis, practice, play, or simply being alive and listening. Before we get to the complexity of "understanding," however, we first need to look at looking and listening and seeing and hearing ${ }^{1}$. To understand musical understanding, we must initially sort out some "typical" questions and try to see what it is we are asking when we ask them. Some obvious candidates are: What, if any, special kind of interest(s) do we take in an artwork or "object"? What is it to

Lawrence D. Kimmel is a professor in the Department of Philosophy of Trinity University. He is the author of Dialectical Convergence-The Imperative of Public Conversation and has contributed essays to Philosophy in Culture and Analecta Husserliana. 
Journal of Aesthetic Education, Vol. 26, No. 3, Fall 1992 
take an artistic interest in any work or object? What are musical looking and listening? Clarity in responding to such questions depends, of course, on getting clear about contexts and contrasts. That is, what other "kinds" of looking, listening, and understanding is musical looking, musical listening, and musical understanding to be compared with and contrasted to? Given this concern, one relevant question is, What is it to listen to music rather than to sounds, or music rather than noise, or music rather than news?

Let's begin on the seemingly simplest seam of it, with the question, When do we listen to sounds? The answer is not "all the time." This may be an answer to the question, When do we hear sounds? After all, it's a noisy world. But listening is something we do, not something that happens. In concert with Scruton's remark that "understanding music is a special case of intentional understanding" ${ }^{2}$ is the point that (musical) listening is a special case of intentional hearing. We can listen with understanding-and sometimes not. We can also listen (but less plausibly, hear) with interest, concern, enjoyment, sympathy, anxiety-or not. To listen is to focus our attention - a kind of focused hearing - in a certain way or in various ways merely suggested above. Consider only the complexity of meaning in the following ordinary complaint of a parent or lover or employer: "Please listen to me! You're not listening, you're not hearing what I say, not understanding what I mean."

Perhaps even in a noisy world it is misleading to say that what we hear all the time are sounds; or perhaps it's simply uninformative-what would it be to hear a nonsound? What we hear are cars, voices, music, birds, radios, songs, bells, horns, wind, echoes, airplanes. There is, in other words, a surface sense in which we hear things, not sounds. If an insistent voice suggests that, speaking accurately, we hear the sounds things make, I will agree. But "sounds-they-make" is a cultural phenomenon too complicated for mere ears. Whatever else this involves, in the light of what we have already shown, this will be a matter of listening and understanding, not simply hearing. To say only, "I am hearing sounds" casts a strange sense on the ordinary matter and manner of hearing. One seldom listens to sounds, simply (not that one listens to sights or feels); one listens, rather, to people, to radios, rain, traffic, trains. . . but to sounds? Only when there is a game or a mystery of some sort - an uncommon absence of identification ("See if you can guess what sound this is...")-is it appropriate to say we listen to sounds. The deep grammatical point to be noted here is that it takes a kind of phenomenological bracketing to talk this way sensibly. I must make an effort to hear your voice as sound or to listen to my radio as sound. The firstorder experience-what is, so to speak, present to mind-is listening to a complex order or structure of things. But isn't this just quibbling, or just word games, as when one insists, "I'm not listening to the radio, I'm listening to the news?" It may be objected that there is really no opposition here, 
that we listen both to the radio, to the news, and to the sounds and that each is simply a different way of focusing on or talking about what is happening. Perhaps this is so, but "simply" won't do. There is a complexity of experience and reference that needs sorting out, a whole range of language games that go with each of the above distinctions. For example, it is the hearing of the news of a stock market crash, of an invasion of a homeland, of a family member's death in an accident that results in the heart attack, the feeling of despair or outrage, not a hearing of sounds. Where, then, is one to focus attention, and why?

II

In these opening remarks I have tried to clarify the direction that inquiry must take toward understanding what it is to hear music. The grammatical complexity of the root distinction between hearing and listening already suggests that the task of inquiry must be to open up and not close down the density of distinctions and choices that present themselves in the pursuit of musical understanding. This background orientation leads to a further question: Is there some particular way of focusing, of attending, that it properly makes sense to call musical? What are we asking here? A prior and subsidiary question that must be asked is what it is we are focusing on when we focus "musically." Is there a general sense or response to the question of what it is we are attending to when we listen to music?

It is not plausible that anyone, anytime, listening to any music attends to the same thing. To begin with, as we have already suggested, the "thing" in question is problematic. At a performance of Boris Godunov one may be listening to the aria, the voice, the orchestra, the chorus, the score, the language; one can and does, that is, listen to many things in listening to one thing. Attending a rare appearance in the United States and hearing Boris Cristov in the role, I may be listening - in listening to the music - to the remarkable resonance of the lower register of a great basso profundo in a bad hall, or to the surprising range and location of the tesitura of the voice, or to the unsettling contrast of his singing in Russian to the Italian of the chorus; and different persons in the audience may be listening to the same thing or not, all the while, with exceptions all listening to the opera. The "identity" of the aesthetic "object" is of course in this way itself problematic. In quite ordinary and other circumstances one may listen to a range of kinds of things - to a voice, or an orchestra, or a program, or a person, or a telegram, or a complaint. There is no exclusive or independently privileged first-order language of sound as such; this, too, is a matter of focus. "First-order language" can and does shift, depending on context, which in turn depends upon interest.

The shift in focus on hearing sound to hearing music is one of a whole 
domain of discourse, a transposition in language games. Consider just the obvious differences in attending to the insipid drone of "elevator music," of "telephone-on-hold music," or the "top twenty-eight," or, perhaps by contrast, to the artistry of Segovia or Hendrix, Horowitz or Gould, Fischer-Discau or Billie Holliday; or, again in a different sense, to solo string or woodwind instruments, to a brass ensemble, to a chamber orchestra, to a cathedral organ, or to a bluegrass banjo. And these examples do not yet raise the complexity of musical form or cultural time and taste. In the latter case, we would need to attend further to the contrast of the compositional styles as well as the musical signatures of Mozart or Mahler, of Bach, Brahms, Bartok, or the Beatles. Musical listening or "attending to" is surely quite different in each of these cases. Things are made even more complicated when we consider whether what we are listening to is "live," recorded, telecast, broadcast, and so on.

Leaving aside questions of judgment - whether of beauty, burlesque, or the banal-consider what experiential differences are involved in listening to the acoustical guitar of Segovia or to the electronic guitar of Hendrix. This is a difference in worlds. In the contrasting voices of Fischer-Discau and Billie Holliday there is a cultural disparity not so difficult to mark between High German and Middle Harlem. Even so, and in some cases, to be able fully to attend musically to one may lead no further; lieder may not sound like music in Harlem, and the anguish of "Strange Fruit" may reach no ear in Hamburg.

A second subsidiary question to the one which begins this section is: What is it to listen to music rather than a series of sounds? This makes it seem as if we could simply set out a series of sounds that are utterly independent of musical sense. We will more profitably focus on "the first move" not of listening to a sound or series of sounds, but to music. If we ask what it is to hear something as music rather than as a series of sounds, the obvious and appropriate concepts or words that rise to fill the gap left by this question are melody, harmony, and rhythm. These by themselves, however, do not explain anything. To hear a sequence of sounds merely, without inclusion in a field of musical understanding, remains just that, a sequence of sounds.

Is it possible to give a neutral description of a series of "musically neutral" sounds? Unless we simply make the sounds, any description other than "Sound 1/Sound 2/Sound 3/Sound N" will carry (semantic) content. That is, any description beyond a series of numbers will suggest dramatic scoring. The correlative point is that unless we already had a musical sense, such distinctions would not carry semantic import. Consider, for example, the following sound distinctions deliberately removed from a defining context of music: 
Jarring sounds / splintering/ muffled / scuffling/ high-pitched sound/

Does this ordinary "nonmusical" sequence already carry sufficient meaning to suggest an interpretation? The further inclusion of barest context shapes it more clearly:

Suddenly there was a jarring noise, like a sharp object being struck against wood. Then, a splintering sound, then muffled voices and scuffling. Then a high-pitched sound, perhaps a scream, then silence.... After a while I fell asleep. I don't know now whether it was a dream.

Here, at any rate, is a sequence of sounds, not music. However, within this sequence is a telling which, even lacking further detail or content, encourages an idea about the kind of interest we might take in the description: "What happened? Who did what to whom? Was the neighboring apartment broken into or not? Should I notify the police? Should I stay out of it? What is my responsibility? What kind of building have I moved into!" We would say perhaps that these kinds of questions indicate more than, or other than, musical interest; but is there a special class of such contrasting interests?

Here is a sequence of sounds no one would mistake for melody, although, by analogy, it is perhaps an orchestration of dramatic events. There is no musical interest yet, certainly no context in which it makes sense to speak of music. But could these sounds be so arranged as to be heard as music? Well, such sounds can be orchestrated; it depends on what the culture has come to regard through tradition, or is willing to accept in innovation, as the stuff of music. Within music history and in contemporary culture, music has been extended and expanded in just this way. (Some would say it has been so adulterated and corrupted.) What, for example, marks the musical distance between the bel canto of Bellini and the shrieks of Berg? Is music or melody "in the ear of the beholder"? It would make better sense to say that music is in the ear (and voice/mind/heart) of the culture. The field of hearing sound as music is set by the culture - the lebenswelt of understanding, part of the fabric of a social, shared sense. Music is not reducible as such to the raw phenomena (the physics and physiology) of mere sound. It is, in its first movement of hearing even, more than sound orchestrated or sound heard as orchestrated. Whatever is heard as music is already organized within a metaphorical framework of movement and structure (melody and harmony), and its existence as sound is marked off in such a way that the relationship is meaningful in time (rhythm).

What and how does this happen? How and when do we learn to "do" all this? Is it the individual mind that must organize sound this way into music? Surely not, or not simply so. In seeking a foundation for aesthetic theory, 


\section{0}

Lawrence D. Kimmel

one might be misled into imagining the necessity of a complex cognitive activity, something that descriptively and factually happens, some procedure that one must do in one's head/mind/brain in order finally to hear music or melody. Listening to music is not, however, a matter of processing discrete bits of sense data. To think so is mistaken in a way detailed by Wittgenstein's criticism of the idea of seeing as the processing of visual sense data. ${ }^{3}$ Recall that on the sense-data theory, seeing has two operative levels: it is something one does after and with a process that first happens, to which one is subject. A "description" of the following sort was offered by Bertrand Russell ${ }^{4}$ to explain what must happen when one sees. Merely in looking and listening one receives data, which one must then convert, so to speak, into things. For the sense-data theorist, seeing an apple thus requires inference.

Without taking up the whole of it here, it is grammatically sensible to say, "I see the apple," but it is grammatically contrived or nonsensical to say, "I see red, round, smooth" and then make some sort of additional movement of synthetic inference and conclude that "this collection of data is probably an apple." This may happen - perhaps in a dark room, with mirrors, in a guessing game - but it would be the exception. We of course may be wrong in making a claim that "I see an apple" or "I hear a melody." We can also imagine a context that allows such a description of ordinary perception (i.e., of seeing colors, not things), but it would be the derivative exception, not the rule.

I may, for example, imagine those red, round, smooth objects scattered in the open field to be burnished stones and then be amazed to see a child pick one up and take a bite out of it; thus a stone becomes an apple. (The familiar analogue in the psychology of perception is that the rabbit becomes a duck.) I now see the objects for what they are, having been mistaken. But in ordinary circumstances, what I see (i.e., the basic units of the language of seeing) are apples, not reds and rounds; they are objects, not datums. So too, in hearing music, it is a melody I hear, not a sequence of sounds. In this most primary sense, musical understanding is something I bring to the experience, not, as in the analogy cited above, a process in which a datum is transformed by inference into a thing.

There is, finally, no one answer to any of the questions we have raised here, and the traditional kinds of answers have seemed to depend on the idea of a musically neutral description of sound. All sound has in it, as sound, the potential and sense of music. This is not to deny that there may be a "first-order" analysis. In the case of the apple it is arguable that the concepts "red, round, hard, smooth" are in some important "visual" sense "first order." But this cannot sensibly preclude an equally plausible analysis that insists that the "first-order" concepts appropriate to the apple are "skin, seeds, pulp, stem, core." Which is more basic, which are the constituants of 
the experience of the apple? This cannot be answered without knowing more of what motivates the question. So too with music: the cultural phenomenon exists first in the "world of appearance," before any arrangement in representation or musical notation.

III

The hearing of music is then, in its first movement of understanding, not a physiological or even psychological process but a cultural faculty or facility. It is misleading at the initial stage of analysis (the first move in the game of understanding music, melody, and tone) to say we hear sequences of sounds (datums of sense) that we then somehow spontaneously, magically, or metaphorically convert to melody and music. There are exceptions surely, where sound is indeterminate, as it were at a distance, we gradually come to distinguish the disturbance as music, as having a melodic, harmonic, or rhythmic character. So, too, in some elemental sense we no doubt gradually learn to hear sound as music within a culture and language in much the same way as we learn language itself: we simply come to hear sounds as words. This does not intend to dismiss the importance of specific questions that may be raised in the context of study. This is Wittgenstein's general point, of course, in the Investigations. If our minds, sense, and spirits remain alive, we continue to learn to hear in new, broader, and deeper ways which are also dependent in complex ways on cultural experience. We can and do move from the experience of the syncopated solo rhythms of the nursery rhyme to the lyrical movements of the ballet, to conversant voices of instruments in orchestral sections; we can move, and as a musically innovative culture have moved, from the tonal wholeness of major keys and harmonic scales to the dynamic structural tensions of minor keys and atonality, from the familiar reassuring tones of dramatic scoring of "classical" works to the distorted pitch of contemporary compositions. We learn to listen and hear in an incredibly complex way; we learn not only what to listen to, but what to listen for in a vast literature and field of music. And if we are fortunate and fascinated, we keep reaming, keep open to the life of sound and to the miracle of musical movement.

But what is it we ream, and how? In the most general terms, we learn as we grow from infancy to speak and listen and think and hear and feel and imagine without a set of directions; there is no clear agreed-upon theory which would begin to account for what is done, much less how it is done. So, too, it is in music. It is not as if we first learn to hear sound and then learn to hear music. It is plausible to say that children may not learn to hear sound at all. For example, when should we say the "occurrence" of sound first begins? To make the point in an extreme (absurd but experientially universal) way, consider for a moment the internal racket of the womb: the 


\section{Lawrence D. Kimmel}

stirring groan of organs going about their metabolic ritual, transforming energy; the strong, steady beat of an organic pump — different, too, in signaling deep sleep or sexual excitement, anxiety or exhaustion. On this account we can imagine a kind of Platonic cave game with sounding shadows in which the fetus spends her sightless time guessing the sounds and sense of passing tone and rhythms. Recall it was a major worry of Plato that the game of guessing shadows would bring such delight and reward that we would never leave the cave. $^{5}$ And so we share, archetypally, the dark, sounding comfort of the womb, which may account later for the phenomenon that "music soothes a savage breast" and, less mythically, that the distressed child may be rocked to sleep by the steady beat of sound.

When does the "learning" of sights and sounds as such begin and how does it occur? These are not empiric questions, I think. Even so, patterning is something rational creatures do naturally; so much so that it is not clear, or perhaps even sensible, to think that there is a requisite theoretical base, a necessary or given ground. By using the word "naturally" here, I intend this whole example in the spirit of Wittgenstein's "remarks on the natural history of Man," and not in any reductive or regressive sense which would reintroduce the physiological as the root of experience, musical or otherwise.

Does our playfully uncovered idea that in the fetal stage the child-to-be "learns" to hear long before it can possibly see mean that the most primitive form of human cognizance is aural rather than visual? What sort of question or claim is this? A nonsensical one, I think, and it may be instructive that it is so. It suggests that in musical understanding there may be no "first move." There may not be a datum within which music is found, out of which music is made-no "given" the transformation of which becomes music. Rather "hearing music" already depends on a broad cultural understanding, not to be reduced to a simple process of conversion or translation. We can, of course, distinguish, as we have herein suggested, the difference between sequence and sound, movement and music. We can show through plausible argument that sequence is not enough to account for melody. Indeed, the metaphor of movement is needed. But this just means that understanding music and accounting for the elementary grammar of it require an intelligible language, a complete language game, an already coherent form of life.

The result of our inquiry is clearly modest. All we have done thus far is to indicate the moveable boundaries of musical sense by showing that the basic unit of analysis is not sound per se or its sequence, but a tonal note and melody within a cultural context of being music. Nor is it clear that there must be some primitive experience of a "given," of isolated sounds that are somehow transposed to sequences, then metaphorically transformed to movement, and, finally, heard as notes of melody. 
We (already) hear music in the same way that we (already) see apples: that is, long before we can begin the anatomic analytic of conceptually isolating "red" and "round" and "smooth" and "sweet" and "hard" and "delicious," or, for that matter, "appealing," "symbolic," and "seductive." Likewise all the complex language of perceiving, appreciating, or "understanding"; the appearance of an apple comes much later than the first step of seeing "apple." A kind of functional counterpoint to any analytic theory of music, even the sensitive and sensible one Scruton offers, is a playful reminder of the sort we have tried to give here: that the audience prior to the prelude, indeed that the baby before birth, already has as part of its sensitivity, the movement and placement of sound, the wonderful, frightening, reassuring rhythm of life, the rudiments of musical understanding of a resonant/dissonant world. However traumatic the advent of first light and air and the rude backsides slap of the attending physician's baton, the child already has, we may surmise, some remembrance on which to draw in the birthing of her own rhythm in a new key and time. Just so does the musical novitiate have the cultural sensibility to pick up and take in full measure the sounds of music. So then are we claiming that the child-to-be listens to the chamber music of the mother's internal organs? No such claim is in order. Still....

It is tempting to break off just here. This would leave it where things actually begin, open to conceptual boundaries set by the simple contextual imperative of making and discovering sense. We can, now far from the trauma of our beginnings, ask all sorts of questions and make all sorts of distinctions, some more helpful or astounding or mysterious and provocative than others. What is it we don't know about listening to music? What is it we wish to make clear to ourselves? Why are we asking the question? It was Wittgenstein's sound counsel that, especially in philosophy, one ought to keep this last question within ready recall.

IV

Have we provided a basis for moving on to the representative questions we set out at the beginning of this article? What is it to take an interest in music? What do we mean by musical interest? Is this a separate phenomenon as well as an analytically distinct category? On the ordinary-language model we have pursued, we can set out examples of contrasting interests: (1) musical interest in contrast to economic interest (Will the record sell? Can we market it?), (2) musical interest in contrast with moral interest (Won't this lyric or performance seduce every Lucy to seek out diamonds in the sky or encourage young musicians to bite bats?), ${ }^{6}$ (3) musical interest in contrast to martial interest (We require the sounding brass and stirring sounds that will lead the ranks of death to their calling in battle), (4) musical interest in contrast 


\section{Lawrence D. Kimmel}

to political interest (What heroic strains will overcome the limp image of presidential wimpery?). Are all of these concerns about musical products or activity not musical? We can perhaps make the case either way, but we need here, as before, a broad cultural field of common understanding and ordinary language to do so.

It is in a similar way that the familiar (Kantian) model of aesthetic theory attempts to purify categories appropriate to art and so defines aesthetic interest as an interest in a visual or auditory phenomenon "for its own sake" — a kind of first-order interest of reflection. And once again, a case may be made herefor example, this may be understood as a proposal that we must first discuss perception as a phenomenon (music) before we can assess or predict its usefulness and impact or effect in an economic, political, or moral context.

Believing this, we may with Nietzsche, following Schopenhauer, support the view that aesthetic or artistic engagement with the world is prior or primary to all else. We can submit the credible notion that we register and respond, assimilate and appropriate the shapes of color and the movement of sound before any imperatives or prescriptions of form and measure direct our management of it. This seems to be, however, a different, metaphysical question: What is it to be grounded in primitive experience, to become aware of the world as such? But even if we locate our interest in "experience" or "world," there is already a cultural structure, mirrored and moored in our shared language, within which our understanding moves. Whatever our response, we can only either describe or propose how it is we wish to use the concepts "musical," "artistic," "interest," and so forth. This description, in turn, depends wholly on the grammar of the concepts, their use in the shared language games of understanding music or painting. The decision that informs any proposal of what musical interest is "really," or the concern such a focus betrays, depends on this: at the very heart of perception, of sense and sensitivity no less than in judgment and theory, is metaphor - or rather, a quite ordinary culturally complex and fictive language of imagination that is everywhere dense and which is an integral part of normal sensibility.

We can, when we choose, sort out, cut up, paste on from the vantage of myriad points, a sensible account of art, just as of science, of experience, of world, of time and space, of meaning and imagination. Is one point more privileged than another, more true or more real? From what point, then, would that question be answered? There is an incredible richness to the fabric of our cultural lives, the acknowledgment of which recommends to aesthetics a moveable focus, not stationary perspective.

Even to focus as we have on musical understanding may make it seem as if the required task of musical aesthetics is to develop a theory to solve puzzles. But which puzzles? They seem endless, and for every puzzle there 
are not too few but too many pieces. Metaphors within metaphors, stacked upon metaphors, all open to the interpretation of philosophy. Just as one cannot perform "music in general" - there is no such thing, its enterprise nonsense-so is there no understanding "music in general." Aside from grammatical tuning of the instruments of inquiry, this makes of musical aesthetics a series of concrete investigations usefully limited to particular cases. If this in turn seems to diminish the interest and impact of aesthetic theory, it also empowers and enriches musical activity.

To mark and make conceptual distinctions between sound and music, sequence and movement, notes and tones, pitch and contrapuntal melody is one among many ways to remark that musical understanding, even in its infancy, is a cultural as well as cognitive task. The moral of the story about music and art is that one learns to love the puzzling process as much as coherent solutions.

Finally, it may be important to remind ourselves that music is surely as much about being moved as about movement, as much about surprise, excitement, joy, suspense, and sorrow as about understanding - which is not to say these are exclusive. The genius of art is in the poesis of its making and remaking, its creation and renewal. We have discussed only beginnings, first movements listening to music, which both presume and promote understanding. There remain open all the ensuing movements in which music moves to mark the fullness of time.

\section{NOTES}

1. I use this locution in the sense of Wittgenstein's counsel in the Blue Book and the Philosophical Investigations, "Don't think-look" (Ludwig Wittgenstein, Philosophical Investigations [New York: Macmillan, 1953], sec. 1, p. 66).

2. Roger Scruton, The Aesthetic Understanding (London: Methuen, 1983), p. 78.

3. See Wittgenstein's discussion of perception and process in Philosophical Investigations, sec. 11, p. xi.

4. The simplest account is provided in Bertrand Russell's The Problems of Philosophy (New York Oxford University Press, 1959), chap. 1.

5. Plato, Republic, Book VII.

6. While the Beatles reference is likely to be familiar, perhaps Ozzie Osborn's early concert antic is not. 